\title{
Conformation, structure, and thermodynamics integrative mechanism related to receptor regulation
}

\author{
Qinyi Zhao* \\ Medical institute, CRRC, Beijing PR China
}

\begin{abstract}
As the proposal of thermodynamic model for protein regulation, we need to give satisfactory explanation to many concepts of receptor regulation. In this paper, many concepts of receptor regulation are interpreted by applying principle of protein thermodynamics and thermodynamic model for protein regulation. The informational integration mechanism among effects of many factors within a receptor is also discussed. It shows that thermodynamic model of receptor regulation can account for all properties of receptor regulation and the studying of protein thermodynamic will benefit for our understanding of receptor regulation.
\end{abstract}

\section{Introduction}

The protein regulation theory has undergone a fundamental change, moving from traditional concept of allosteric regulation, an experimental model which was established on protein threedimensional structure theory, to concept of allodynamic regulation, a theoretical model which was established on protein thermodynamic structure theory [1-4]. The thermodynamic theory of protein regulation has integrated many concepts of protein dynamics, protein thermodynamics, protein thermodynamic structure into a holonomic model [4-6]. In this critical moment, we need to recheck traditional scientific terms, concept, basic concepts, facts, logic, and others. In this paper, we have given new explanation to many concepts of receptor regulation based on thermodynamic model for protein regulation. Within this revised model, the thermodynamic mechanisms of information integration of effects of many regulators within a receptor are discussed.

To do it, we need more experimental data and to measure many types of distribution curves of active conformation of a receptor, but there is only a few studying in fields of receptor and. Fortunately, we have gotten more such data and curves in field of enzymology and we have theoretically shown that general profile of conformational distribution curves is similar for near all enzymes and receptors, thus, we can analyze properties of receptor regulation data obtained from enzymology $[1,4,6]$.

\section{General concepts}

Protein thermodynamic structure theory, a protein is not the uniform of a thermal system, but composed of many sub-systems of thermodynamics; any biological function or property of a protein is logically corresponding to a sub-system of thermodynamics within a protein. This sub-system of thermodynamics also represents an ensemble of protein conformations at atom level [7,8]. By applying these principles, we have pictured some types of protein conformation ideal fashion of receptor regulation [3].
In Figure 1, we have drawn up several types of protein conformation involving in receptor regulation. A receptor has many regulatory sites.

The active conformation refers to protein conformation at advanced or global level. The regulatory conformation at regulatory site refers to local conformation, but it also represents an ensemble of protein conformation at atom level $[7,8]$.

Within simple model of receptor activation or two state theory, the agonist binds active conformation more tightly than inactive conformation [9-11]. This is no such concept within our model. Within our theory, the agonist binds to regulatory site of a receptor,

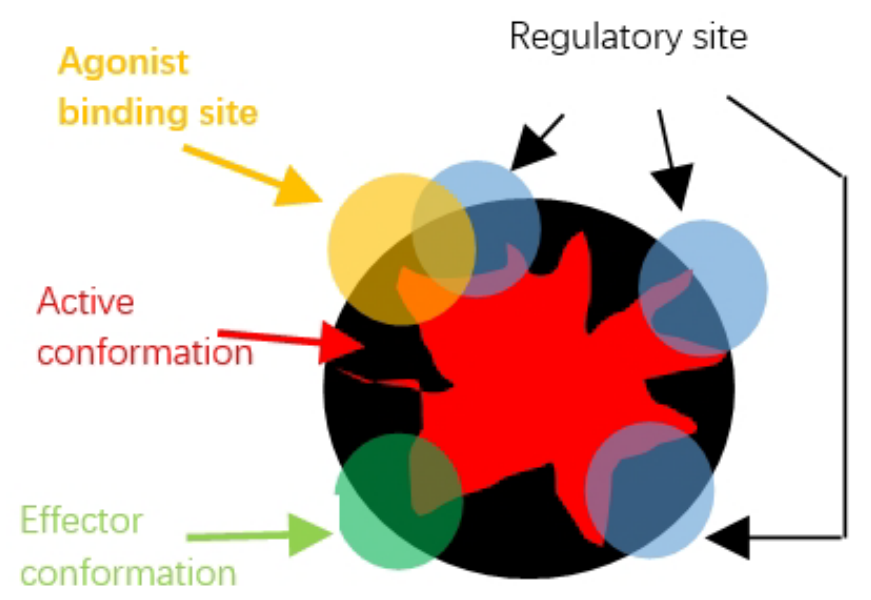

Figure 1. Ideal protein thermodynamic structure of a receptor

Correspondence to: Qinyi Zhao, Medical institute, CRRC, Beijing PR China, E-mail: qinyizhao@gmail.com

Key words: receptor activation, informational integration, thermodynamics

Received: August 10, 2017; Accepted: September 08, 2017; Published: September 11, 2017 
the binding conformation between it and receptor represents a local protein conformation and it couples or overlaps to active conformation of the receptor, and the change of it can be transmitted into active conformation. By this way, it alters the properties of thermodynamic state of active conformation of the receptor and then can regulate or modulate the probability of active conformation of the receptor, this finally results in the change of receptor activity. By applying principle of protein thermodynamic structure theory and thermodynamics, following conclusions could be obtained.

\section{Binding affinity between agonists and receptor}

The binding affinity between a receptor and agonist is a prerequisite for agonists activity, but it is not satisfactory condition for agonist activity. When an agonist binds to an area near to regulatory site of a receptor, it can induce the protein conformational change at this area. And conformation of regulator binding site can modulate the probability of active conformation of a receptor. As the binding site between agonist and receptor is not same to that of regulatory site, the binding affinity is not directly related to potency of an agonist.

\section{Potency of an agonist}

Theoretically speaking, the protein conformational state at regulatory site represents an ensemble of protein conformation at atom level. Different conformations at regulatory site couple with differently conformation of a receptor and have different ability to regulate probability of active conformation of the receptor. agonists can change of the properties of regulatory conformation of a receptor, and different agonists have different potency.

\section{Agonists as positive regulator}

Within our theory, the agonist is a member of positive regulator, and they all increase the probability of active conformation of a receptor via same mechanism. They differ from some aspects: normally, the agonist is endogenous, and without the activation induced by agonist, the receptor remains in inactive state, but for regulator, the case is different.

\section{Thermodynamic integration among agonist, receptor and environmental factor}

The interaction among agonist, receptor and environmental factor can be analyzed by distribution curves of active conformation of the receptor along a quantitative gradient of the environmental factor, such as temperature, $\mathrm{pH}$. When a regulator binds to a receptor, the thermodynamic properties of all types of protein conformations of a receptor are changed the thermodynamic integration of results in the alteration of distribution curve of active conformation of receptor regulator complex is changed when compared to naked receptor. At present, there is no method which can be utilized to measure thermodynamic parameters of protein conformation of a receptor experimentally, but we can diagnose it by a change of distribution curve of active conformation of a receptor-regulator complex when compared with that of naked receptor. The normal profile and principle of it is shown in Figure 2.

In Figure 2, we see that the optimum activity of a receptor with highest probability of active conformation occurs at one condition. We can get following conclusions by studying this Figure.

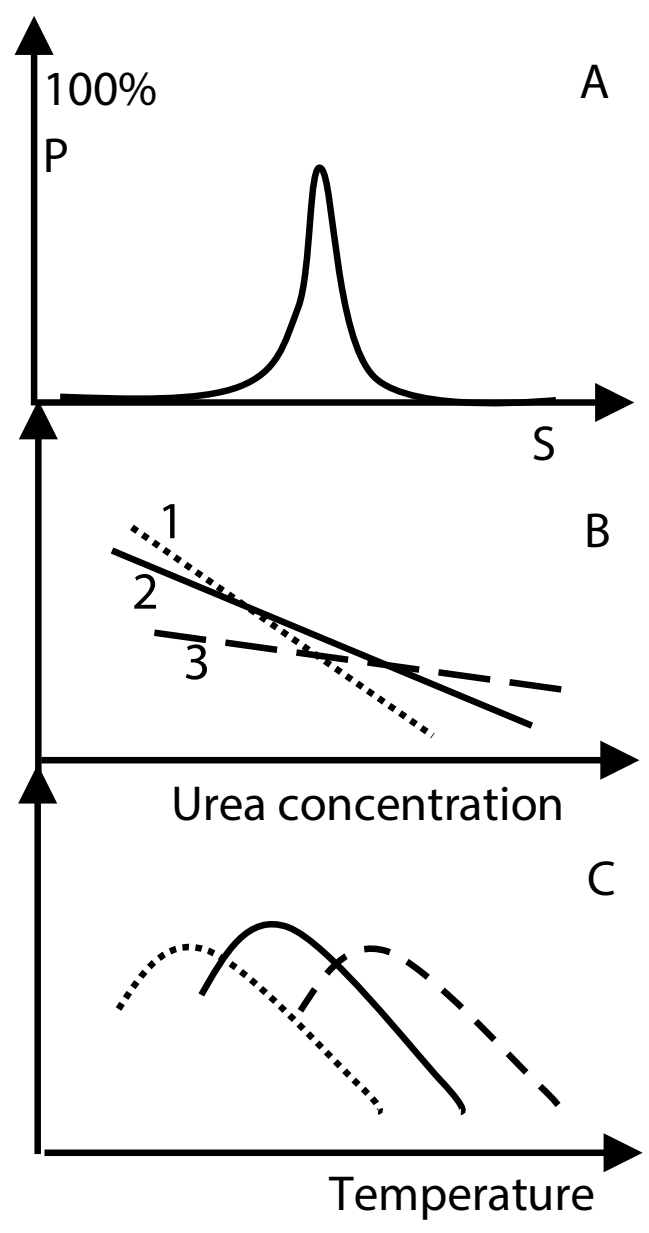

Figure 2. The distribution curve of active conformation of the receptor $\mathrm{P}$ is probability of active conformation of the receptor-regulator complex, $s$ is the value for quantifying an environmental factor, such as temperature, urea, or salt concentration of the buffer, $\mathrm{pH}$. Figure $2 \mathrm{~A}$ is distribution curve of active conformation of the receptor. Figure $2 \mathrm{~B}$ and $2 \mathrm{C}$ is the protein conformation stability curves of the receptor. Line2 represent active conformation. line 1 and line 3 represent inactive conformation. (Figure $2 \mathrm{~B}$ and $2 \mathrm{C}$ is not experimental data, we figure it according to general principles of protein conformation stability, see ref. 6 ). Both figure $2 \mathrm{~B}$ and $2 \mathrm{C}$ can give same distribution curve of active conformation of a receptor as expressed in figure $2 \mathrm{~A}$.

\section{Full agonist and partial agonist}

The potency of an agonist is changeable with a changing condition, a partial agonist measured under one condition can become full agonist under another condition and vice versa.

\section{Antagonists and agonists}

An agonist can behave antagonist under different conditions. Normally, we test agonist at one condition, but at other conditions, it may act antagonist.

\section{Positive and negative regulator}

The effect of regulator is changeable and a positive regulator can become negative agonist in a different condition. But it is not the rule.

\section{Agonist as protein conformation denaturant}

In optimum condition under which a receptor show no activity and receptor-agonist show highest activity, the agonist acts protein denaturant for resting conformation of the receptor. 


\section{Thermodynamic integration among different regulators}

The interaction or the informational integration among many regulator factors can also be analyzed by distribution curve of active conformation of a receptor. Here, the gene mutation, protein modification act regulators of the receptor and they have great impact on the stability of protein conformation. Again, the integration of many factors on protein conformation stability can be monitored by a change of the shape and positions of distribution curve of active conformation of a receptorregulators complex. We have pictured a typical case for that as follows.

In Figure 3, we see that an agonist -receptor complex shows different activity under a given condition. The gene mutation of a receptor can alter the protein conformation stability (and protein stability) and thus adjust receptor activity [12].

For interaction among many different regulators on receptor with similar stability, we can analyze it in Figure 4.

In green area, both regulators are increasing protein dynamics of the receptor and the increasing of protein flexibility can increase receptor activity, the cooperation between effects of two regulators can further increase protein dynamics (or flexibility) of the receptor and this results in high receptor activity.

In gray area, as probability of active conformation of one receptorregulator complex has reached $100 \%$, the cooperation between regulators on receptor regulation will not appear. In this case, the interaction between regulators on protein conformation stability still occur, but it will not be expressed in receptor activity.

If one regulator induced a dramatical change of protein stability of a receptor and another regulator has little impact on it, then the regulator with high impact on protein stability will dominate the interaction among different regulators. The principle can be clearly seen in Figure 5.

In this Figure 5, we see that regulator 1 has great impact on receptor stability and in the interaction between regulator 1 and regualtor2, the regualtor 1 will dominate such interaction.

This is the thermodynamic mechanism for inverse agonist (as regualtor1 in Figure5). The inverse agonist alone acts positive regulator for a receptor, but as negative regulator for a constitutive receptor with full activity. Thus, we predict that the inverse agonist-receptor complex should be more stable than that of receptor with constitutive $[13,14]$.

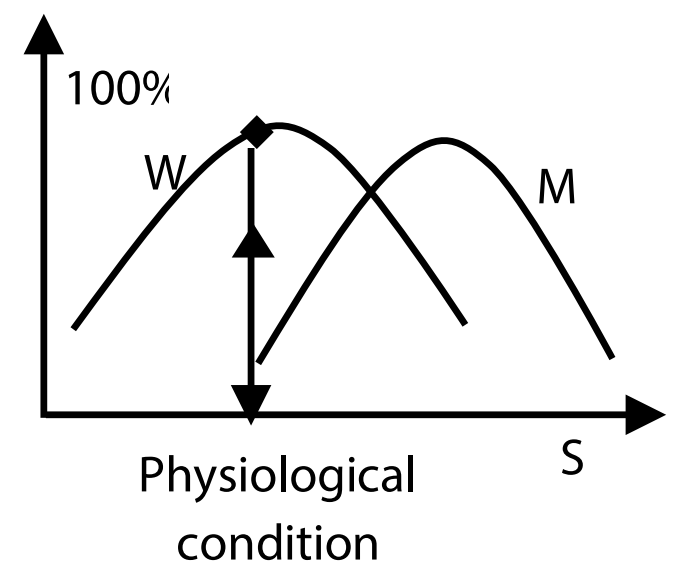

Figure 3. Diagram for receptor regulation by gene mutation $\mathrm{W}$ is distribution curve of active conformation of wild type receptor-agonist complex, $\mathrm{M}$ is distribution curve of active conformation of mutant receptor. $\mathrm{s}$ is the value for quantifying an environmental factor. At a given condition, the receptor activity is altered by gene mutation.

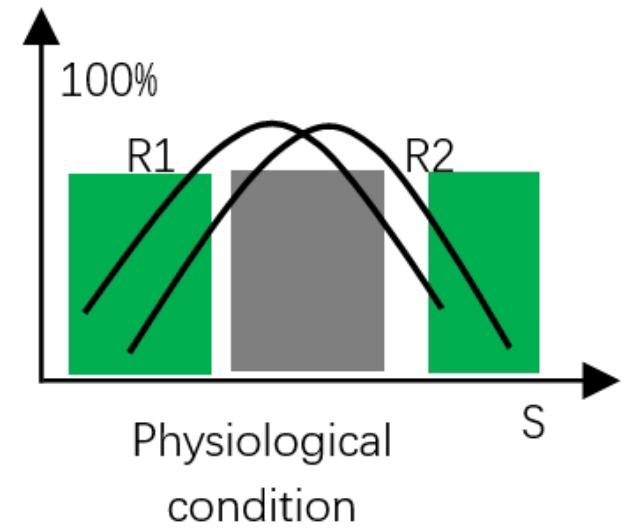

Figure 4. Integration of effects of two regulators within a receptor R1 is distribution curve of active conformation of wild type receptor-regulator 1 complex, $\mathrm{R} 2$ is distribution curve of active conformation of receptor-regulator 2 complex. $s$ is the value for quantifying an environmental factor, such as temperature and urea concentration.

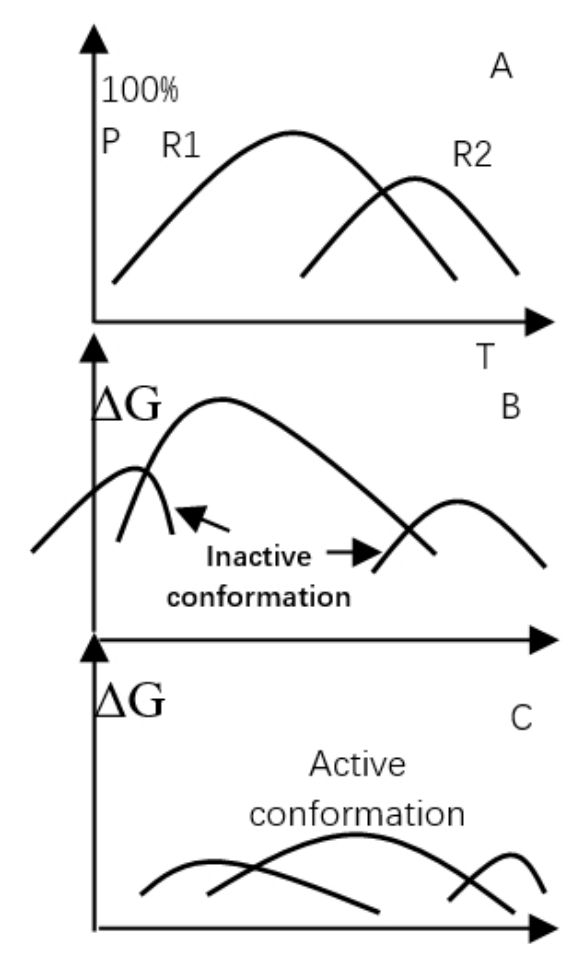

Figure 5. Diagram of stability and regulators interaction. Figure $5 \mathrm{~A}$ is distribution curve of active conformation of a receptor complex with regulator 1 and 2. Figure $5 \mathrm{~B}$ is stability curves (active conformation and two types of inactive conformation) of receptor-regulator1 complex. Figure $5 \mathrm{C}$ is stability curves of receptor regulator 2 complex.

\section{Thermodynamics integration among effects of environmental factors within a receptor}

Some types of physical factors, such as temperature, and chemical substance, (including volatile anesthetics, urea and protein conformation denaturant), take their effects on receptor regulation as environmental factors. Their effect on receptor activity by protein flexibility or dynamics. Within our model, they all influence protein conformation stability of a receptor. In this case, the thermodynamics integration among these factors become very simple. Their effects on receptor activity can be predicted from their properties and knowledge of protein stability. 
In anesthesiology, different volatile anesthetics can be freely combined and their effects on anesthesia induction are additive $[15,16]$. The high pressure can neutralize the effect for volatile anesthetics for they have opposite effect on protein dynamics and stability [17].

Over all, the thermodynamic mechanism for the interaction of regulators on receptor can account for all phenomena we have known. The studying of distribution curve of active conformation of the receptor will enhance our understanding of drug mechanism and interaction among them, as well as working mechanism of receptor regulation.

\section{References}

1. Zhao Q (2015) A thermodynamic and theoretical view for enzyme regulation. Biochemistry (Mosc) 80: 1-7. [Crossref]

2. Zhao Q (2013) Allodynamic Regulation of Protein Activity. Quantum Matter 2: 144 152

3. Zhao Q (2016) Classification of Enzyme Regulators within Thermodynamic Model of Enzyme Regulation. Mol Enz Drug Tar 2: 14

4. Zhao Q (2017) On the indirect relationship between protein dynamics and enzyme activity. Prog Biophys Mol Biol 2017 125:52-60

5. Zhao Q (2009) Protein thermodynamic structure. IUBMB Life 61: 600-606. [Crossref]

6. Zhao Q (2012) partition function of protein conformational state. J Comput Theor Nanosci 9: 745-751

7. Shaw DE, Maragakis P, Lindorff-Larsen K, Piana S, Dror RO, et al. (2010) Atomiclevel characterization of the structural dynamics of proteins. Science 330: 341-346.
8. DePristo MA, de Bakker PI, Lovell SC, Blundell TL (2003) Ab initio construction of polypeptide fragments: efficient generation of accurate, representative ensembles. Proteins: Structure, Function, and Bioinformatics, 5, 41-55. [Crossref]

9. Leff P (1995) The two-state model of receptor activation. Trends Pharmacol Sci 16 89-97. [Crossref]

10. Kenakin, T. (2001) Inverse, protean, and ligand-selective agonism: matters of receptor conformation. The FASEB J 15(3): 598-611. [Crossref]

11. Samama P, Pei G, Costa T, Cotecchia S, Lefkowitz RJ (1994) Negative antagonists promote an inactive conformation of the beta 2 -adrenergic receptor. Mol pharmacol 45(3): 390-394. [Crossref]

12. Gether U, Ballesteros JA, Seifert R, Sanders-Bush E, Weinstein H, et al. (1997) Structural Instability of a Constitutively Active G Protein-coupled Receptor agonistindependent activation due to conformational flexibility. J Biol Chem 272: 2587-2590. [Crossref]

13. Zocher M, Fung JJ, Kobilka BK, Müller DJ. (2012) Ligand-specific interaction modulate kinetic, energetic, and mechanical properties of the human $B 2$ adrenergic receptor. Structure 20: 1391-1402. [Crossref]

14. Wijayaratne AL, McDonnell DP (2001) The human estrogen receptor-a is ubiquitinated protein whose stability is affected differentially by agonists, antagonists, and selective estrogen receptor modulators. JBC 276: 35684-35692. [Crossref]

15. Jenkins A, Lobo IA, Gong D, Trudell JR, Solt K, et al. (2008) General anesthetics have additive actions on three ligand-gated ion channels. Anesth Analg 107(2): 486. [Crossref]

16. Wang A, Zhang Z, Zhao Q (2009) In vivo measurement of protein functional changes. Int J Biol Sci 5: 411-420. [Crossref]

17. Franks NP, Lieb WR (1994) Molecular and cellular mechanisms of general anaesthesia. Nature 367: 607-614. [Crossref]

Copyright: $(02017$ Zhao O. This is an open-access article distributed under the terms of the Creative Commons Attribution License, which permits unrestricted use, distribution, and reproduction in any medium, provided the original author and source are credited. 